Bull. Austral. Math. Soc.

Vol. $42(1990) \quad[437-446]$

\title{
NONSMOOTH INVEXITY
}

\section{Thomas W. ReILAND}

The concept of invexity is extended to nondifferentiable functions. Characterisations of nonsmooth invexity are derived as well as results in unconstrained and constrained optimisation and duality. The principal analytic tool is the generalised gradient of Clarke for Lipschitz functions.

\section{INTRODUCTION}

The needs of optimisation theory have served as the catalyst for the development of an important new class of functions known as invex functions. In inequality constrained optimisation the classical Kuhn-Tucker necessary conditions are also sufficient for optimality if the functions delimiting the problem are convex or satisfy certain generalised convexity properties such as pseudo-convexity or quasi-convexity. The concept of invexity generalises the notion of convexity and is particularly interesting from an optimisation viewpoint since it provides a broader setting in which the Kuhn-Tucker conditions are sufficient for optimality. Hanson [9] was the first to define the concept which would eventually be termed invexity when he considered a differentiable function $f: R^{n} \rightarrow R$ for which there exists $\eta(x, u) \in R^{n}$ such that

$$
f(x)-f(u) \geqslant[\nabla f(u)]^{T} \eta(x, u) ;
$$

note that if $f$ is convex, then $\eta(x, u)=x-u$ can be chosen to satisfy (1). The utility of functions satisfying (1) was quickly established when Hanson showed that if the objective and constraint functions of a nonlinear programming problem satisfy (1) for the same $\eta$, then weak duality and the sufficiency of the Kuhn-Tucker conditions still hold. Applications to optimisation were advanced further when invex functions were shown to have the following fundamental property, first shown by Craven and Glover [7] in an infinite-dimensional setting (for a simple proof in finite dimensions, see Ben-Israel and Mond [1]):

THEOREM 1. The function $f$ is invex if and only if every stationary point is a global minimum.

Hanson's initial results inspired a great deal of subsequent work which has greatly expanded the role of invexity in optimisation. Craven $[4,5]$ and Mond and Hanson

Received 12 December 1989

Copyright Clearance Centre, Inc. Serial-fee code: 0004-9729/90 \$A2.00+0.00. 
[17] extended (1) to include cones. Craven [5] also coined the term invex (for invariant convex) when he showed that $f$ satisfies (1) when $f=h \circ \phi$, where $h$ is convex and $\phi$ is differentiable with $\phi^{\prime}$ having full rank (convexity is not preserved in this situation), and provided second order sufficient conditions for invexity. Conditions necessary or sufficient for cone-invexity were given in Craven [5] and Hanson and Mond [11] while Craven and Glover [7] characterised cone-invex functions defined on abstract spaces in terms of Lagrange multipliers and presented classes of cone-invex functions. Other forms of invexity have also been introduced; Martin [15] defines Kuhn-Tucker invexity and weak duality invexity, while Jeyakumar [14] speaks of strong and weak invex functions. Hanson and Mond [10] formulated a type of generalised convexity by introducing a sublinear functional on the right-hand side of (1) and used it to establish duality between a nonlinear programming problem and its Wolfe dual; Egudo and Mond [8] subsequently used Hanson-Mond generalised convexity to establish duality between a nonlinear programming problem and its Mond-Weir dual (Mond and Weir [18]). Hanson and Mond [11] define Type $I$ and Type $I I$ functions and obtain results concerning optimality conditions, duality, and converse duality for a primal problem and the corresponding Wolfe dual.

For the most part, the study of invexity has been in the context of differentiable functions. Exceptions are Craven and Glover [7], where invexity for quasidifferentiable functions is characterised in terms of Lagrange multipliers and optimality and duality results are obtained, and Craven [6], where a generalised invex condition is imposed on a (not necessarily differentiable) Lipschitz function to obtain a weak duality result.

In this paper we further extend invexity to the nondifferentiable setting by defining invexity for Lipschitz functions. We generalise many of the results concerning properties of invex functions, optimality conditions and duality that have been established in the differentiable case. Our principal analytic tool is the generalised gradient of Clarke [2], the salient features of which are reviewed in the next section. In Section 3 we present properties of invex Lipschitz functions and study unconstrained optimisation, while in Section 4 we investigate constrained minimisation problems and duality.

\section{Technical preliminaries}

In this section we introduce the generalised gradient of a Lipschitz function and related concepts; the results needed in the sequel are presented without proof. For a more complete treatment of the theory, motivation, and application of generalised gradients and extensive references we refer the reader to Clarke [3].

Let $X_{0}$ be an open subset of $R^{n}$; the function $f: X_{0} \rightarrow R$ is Lipschitz on $X_{0}$ if there exists a positive constant $k$ such that

$$
\left|f\left(x_{1}\right)-f\left(x_{2}\right)\right| \leqslant k\left\|x_{1}-x_{2}\right\| \text { for all } x_{1}, x_{2} \in X_{0} .
$$


Definition 1: If $f: X_{0} \rightarrow R$ is Lipschitz on $X_{0}$, the generalised directional derivative of $f$ at $x \in X_{0}$ in the direction $v \in R^{n}$, denoted $f^{0}(x ; v)$, is given by

$$
f^{0}(x ; v)=\underset{\substack{y \rightarrow x^{x} \\ \lambda \downarrow 0}}{\limsup } \lambda^{-1}[f(y+\lambda v)-f(y)] .
$$

By the Lipschitz condition it follows that $\left|f^{0}(x ; v)\right| \leqslant k\|v\|$, so $f^{0}(x ; v)$ is a welldefined finite quantity. As a function of $v, f^{0}(x ; v)$ is Lipschitz on $X_{0}$, subadditive, that is, $f^{0}\left(x ; v_{1}+v_{2}\right) \leqslant f^{0}\left(x ; v_{1}\right)+f^{0}\left(x ; v_{2}\right)$, and positively homogeneous, that is, $f^{0}(x ; \lambda v)=\lambda f^{0}(x ; v)$ for $\lambda \geqslant 0$. The latter two properties permit the following definition of the generalised gradient.

Definition 2: The generalised gradient of $f$ at $x \in X_{0}$, denoted $\partial f(x)$, is defined as follows:

$$
\partial f(x)=\left\{\xi \in R^{n}: f^{0}(x ; v) \geqslant \xi^{T} v \text { for all } v \in R^{n}\right\} .
$$

If $x \in X_{0}$ is such that $0 \in \partial f(x)$, then $x$ is said to be a stationary point of $f$. The following theorem summarises some of the fundamental results concerning $\partial f(x)$.

TheOREM 2. (Clarke [3]) (a) $\partial f(x)$ is a nonempty, convex, compact subset of $R^{n}$ and $\|\xi\| \leqslant k$ for every $\xi \in \partial f(x)$.

(b) For any $v \in R^{n}$,

$$
f^{0}(x ; v)=\max \left\{\xi^{T} v: \xi \in \partial f(x)\right\} .
$$

(c) If $f$ is continuously differentiable at $x \in X_{0}$, then $\partial f(x)=\{\nabla f(x)\}$. If $f$ is convex, then $\partial f(x)=\partial_{c} f(x)$, where $\partial_{c}$ denotes the subdifferential of convex analysis. For all Lipschitz functions $f, \partial f(x)=\partial_{c} f^{0}(x ; 0)$.

(d) $\partial(-f)(x)=-\partial f(x)$; if $g: X_{0} \rightarrow R$ is Lipschitz on $X_{0}$, then

$$
\partial(f+g)(x) \subseteq \partial f(x)+\partial g(x)
$$

(e) Let $D_{f}$ be the set of points in $X_{0}$ at which $f$ is not differentiable (by Rademacher's Theorem (Stern [20]) $D_{1}$ has Lebesgue measure zero) and let $S$ be any other set of measure zero in $R^{n}$. Then

$$
\partial f(x)=\operatorname{co}\left\{\lim _{k \rightarrow \infty} \nabla f\left(x_{k}\right): x_{k} \rightarrow x, x_{k} \notin S \cup D_{f}\right\} ;
$$

that is, $\partial f(x)$ is the convex hull of all points of the form $\lim \nabla f\left(x_{k}\right)$, where $\left\{x_{k}\right\}$ is any sequence which converges to $x$ and avoids $S \cup D_{f}$.

(f) For $D_{f}$ and $S$ as in (e),

$$
f^{0}(x ; v)=\underset{y \rightarrow x}{\limsup }\left\{[\nabla f(y)]^{T} v: y \notin S \cup D_{f}\right\} .
$$


For $f: R^{n} \rightarrow R, f^{\prime}(x ; v)$ denotes the directional derivative of $f$ at $x$ in the direction $v$, where

$$
f^{\prime}(x ; v)=\lim _{\lambda \downarrow 0} \lambda^{-1}[f(x+\lambda v)-f(x)]
$$

when this limit exists. If a Lipschitz function $f$ has a directional derivative, it is not necessarily true that $f^{\prime}(x ; v)=f^{0}(x ; v)$; if for all $v$ the directional derivative $f^{\prime}(x ; v)$ exists and equals $f^{0}(x ; v)$, the Lipschitz function $f$ is said to be regular at $x$. If $g: X_{0} \rightarrow R$ has a directional derivative at $x$ for each $x \in X_{0}, g$ is quasidifferentiable if there exists a convex compact subset $M(x)$ of $R^{n}$ such that for each $x \in X_{0}$ and every $v \in R^{n}$,

$$
g^{\prime}(x ; v)=\max \left\{\xi^{T} v: \xi \in M(x)\right\} .
$$

If $g: X_{0} \rightarrow R$ is quasidifferentiable and the multifunction $M(\cdot)$ is upper semicontinuous, then $g$ is a regular Lipschitz function on $X_{0}$ with $M(x)=\partial f(x)$ (Clarke [2]).

\section{NONSMOOTH INVEXITY AND UNCONSTRAINED OPTIMISATION}

Definition 3: Let $f: X_{0} \rightarrow R$ be Lipschitz on $X_{0}$, where $X_{0}$ is an open subset of $R^{n}$; then $f$ is invex on $X_{0}$ if there exists a function $\eta: X_{0} \times X_{0} \rightarrow R$ such that

$$
f(x)-f(u) \geqslant f^{0}(u ; \eta(x, u)) \text { for all } x, u \in X_{0} .
$$

Craven and Glover [7] define invexity for a quasidifferentiable function by substituting $f^{\prime}(u ; \eta(x, u))$ for $f^{0}(u ; \eta(x, u))$ in (2). Note that if $f$ is quasidifferentiable and the multifunction $M(\cdot)$ is upper semicontinuous, then if $f$ is invex according to Craven and Glover it is invex according to Definition 3.

The next theorem extends to the nondifferentiable case a result found in Ben-Israel and Mond [1] and Craven and Glover [7].

Theorem 3. Let $f: X_{0} \rightarrow R$ be Lipschitz on $X_{0}$ with $X_{0} \subseteq R^{n}$ open. For each $u \in X_{0}$, assume that for every $x \in X_{0}$ the convex cone

$$
K_{x} \equiv \bigcup_{\lambda \geqslant 0}(\lambda \partial f(u) \times\{\lambda(f(x)-f(u))\})
$$

is closed. Then $f$ is invex on $X_{0}$ if and only if every stationary point is a global minimum of $f$ over $X_{0}$.

Proof: $(\Rightarrow)$ Suppose $f$ is invex and $0 \in \partial f(u)$; then $f^{0}(u ; v) \geqslant 0$ for all $v \in R^{n}$ by definition of $\partial f(u)$, which implies $f(x)-f(u) \geqslant 0$ for all $x \in X_{0}$.

$\Leftrightarrow$ Suppose every stationary point is a global minimum of $f$ over $X_{0}$. If $0 \in$ $\partial f(u)$, choose $\eta(x, u)=0$ in Definition 3. Suppose $0 \notin \partial f(u)$. If $x=u$, then 
$(0,-1) \notin K_{x}$; if $x \neq u$ and $(0,-1) \in K_{x}$, there exists $\lambda \geqslant 0$ such that $0 \in \lambda \partial f(u)$ and $\lambda(f(x)-f(u))=-1$. If $\lambda>0$, then $0 \in \partial f(u)$, which contradicts $0 \notin \partial f(u)$, and if $\lambda=0$, then $0=-1$. Thus $(0,-1) \notin K_{z}$ for all $x \in X_{0}$, and by Theorem 2(c) and a theorem of the alternative, $[6$, Theorem 7], there exists $\eta=\eta(x, u)$ such that $f(x)-f(u) \geqslant f^{0}(u ; \eta(x, u))$.

Corollary 1. Let $f: X_{0} \rightarrow R$ be invex on $X_{0}$. Then $u \in X_{0}$ is a global minimum of $f$ over $X_{0}$ if and only if $0 \in \partial f(u)$.

Proof: Since $X_{0}$ is open, necessity follows. Sufficiency follows directly from the first part of the above proof.

COROLlary 2. If $f: X_{0} \rightarrow R$ has no stationary points and the convex cone $K_{x}$ is closed, then $f$ is invex on $X_{0}$.

Straightforward extensions of (2) can be made as follows:

$$
f^{0}(u ; \eta(x, u)) \geqslant 0 \Rightarrow f(x)-f(u) \geqslant 0,
$$

and

$$
f(x)-f(u) \leqslant 0 \Rightarrow f^{0}(u ; \eta(x, u)) \leqslant 0 .
$$

Functions satisfying (4) and (5) for all $x, u \in X_{0}$ will be said to be, respectively, pseudoinvex on $X_{0}$ and quasi-invex on $X_{0}$. Choosing $\eta(x, u)=x-u$ in (4) shows that the pseudo-convex functions in Reiland [19] are pseudo-invex. The pseudo-invex and quasiinvex functions of Hanson [9] defined for the differentiable case are special cases of (4) and (5), respectively. If $f: X_{0} \rightarrow R$ is invex on $X_{0}$ then $f$ is both pseudo-invex and quasi-invex on $X_{0}$. Conversely, if $f$ is pseudo-invex on $X_{0}$ and satisfies (3), then by Theorem $3 f$ is invex on $X_{0}$ since if $u$ is a stationary point of $f,(4)$ implies $u$ is a global minimum of $f$ over $X_{0}$. Thus, for the class of (Lipschitz) functions satisfying (3), pseudo-invex and invex on $X_{0}$ are equivalent. Another special case of pseudo-invexity is the concept of semi-convex, defined by Mifflin [16] for regular Lipschitz functions $f: X_{0} \rightarrow R$ as follows: $f$ is semi-convex at $u \in X_{0}$ if for every $d \in R^{n}$ such that $u+d \in X_{0}, f^{\prime}(u ; d) \geqslant 0 \Rightarrow f(u+d) \geqslant f(u)$. If $f$ is semi-convex at $u$ for every $u \in X_{0}$, then choosing $d=x-u$, where $x \in X_{0}$, shows that $f$ is pseudo-invex on $X_{0}$ with $\eta(x, u)=x-u$.

We conclude this section with a result that gives a sufficient condition for invexity.

THeOREM 4. If $f: X_{0} \rightarrow R$ is Lipschitz on $X_{0}$ and for each $x, u \in X_{0}$ there exists a neighbourhood $N(u)$ of $u$ and $\eta(x, u) \in R^{n}$ such that

$$
f(y+\lambda \eta(x, u)) \leqslant \lambda f(x)+(1-\lambda) f(y), \text { for all } y \in N(u) \text { and } 0 \leqslant \lambda \leqslant 1,
$$


then $f$ is invex on $X_{0}$.

Proof: Let $\lambda_{n}^{-1}\left[f\left(u_{n}+\lambda_{n} \eta(x, u)\right)-f\left(u_{n}\right)\right]$ be a sequence converging to $f^{0}(u ; \eta(x, u)) \equiv \limsup _{\substack{y \rightarrow u \\ \lambda \downarrow 0}} \lambda^{-1}[f(y+\lambda \eta(x, u))-f(y)]$, where $\lambda_{n}$ is a positive sequence of real numbers converging to 0 and $\left\{u_{n}\right\} \rightarrow u$. Then

$$
\begin{aligned}
f^{0}(u ; \eta(x, u)) & =\lim _{n \rightarrow \infty} \lambda_{n}^{-1}\left[f\left(u_{n}+\lambda_{n} \eta(x, u)\right)-f\left(u_{n}\right)\right] \\
& \leqslant \lim _{n \rightarrow \infty}\left\{f(x)-f\left(u_{n}\right)\right\} \\
& =f(x)-f(u) .
\end{aligned}
$$

REMARK. If $f$ is differentiable then the hypothesis in the above theorem can be weakened to $f(u+\lambda \eta(x, u)) \leqslant \lambda f(x)+(1-\lambda) f(u), 0 \leqslant \lambda \leqslant 1$, and the result is then identical to Theorem 2 in Ben-Israel and Mond [1].

\section{Constrained optimisation and duality}

Consider the inequality constrained nonlinear programming problem

$$
\operatorname{Min}_{x \in X_{0}} f(x) \text { such that } g_{i}(x) \leqslant 0, i=1, \ldots, m \text {, }
$$

where $X_{0} \subseteq R^{n}$ is open and $f, g_{i}: X_{0} \rightarrow R, i=1, \ldots, m$, are Lipschitz on $X_{0}$. A point $a \in R^{n}$ is feasible for (P) if $a \in X_{0}$ and $g_{i}(a) \leqslant 0, i=1, \ldots, m$. If $x_{0} \in X_{0}$ is a local minimum for $(P)$ and a constraint qualification is satisfied, then by Hiriart-Urruty [12, Theorem 6] the following generalised form of the Kuhn-Tucker conditions hold:

$$
\begin{aligned}
& 0 \in \partial f\left(x_{0}\right)+\sum_{i=1}^{m} \lambda_{i} \partial g_{i}\left(x_{0}\right) ; \\
& \lambda_{i} g_{i}\left(x_{0}\right)=0, i=1, \ldots, m ; \\
& \lambda_{i} \geqslant 0, i=1, \ldots, m .
\end{aligned}
$$

The following theorem shows that if the functions delimiting $(P)$ are invex with respect to a common $\eta$, then (5)-(7) are also sufficient for optimality.

THEOREM 5. Suppose $x_{0}$ is feasible for $(P)$ and that the Kuhn-Tucker conditions (5) - (7) are satisfied at $x_{0}$. If $f$ and $g_{i}, i=1, \ldots, m$, are invex on $X_{0}$ for the same $\eta$, then $x_{0}$ is a global minimum of $(P)$.

Proof: Let $x$ be any other feasible point for (P); then

$$
\begin{aligned}
f(x)-f\left(x_{0}\right) \geqslant & f(x)+\sum_{i=1}^{m} \lambda_{i} g_{i}(x)-f\left(x_{0}\right)-\sum_{i=1}^{m} \lambda_{i} g_{i}\left(x_{0}\right), \\
& \quad \text { by feasibility of } x \text { and }(6), \\
\geqslant & f^{0}\left(x_{0} ; \eta\left(x, x_{0}\right)\right)+\sum_{i=1}^{m} \lambda_{i} g_{i}^{0}\left(x_{0} ; \eta\left(x, x_{0}\right)\right) ;
\end{aligned}
$$


by (5) there exists $\xi \in \partial f\left(x_{0}\right)$ and $\xi_{i} \in \partial g_{i}\left(x_{0}\right), i=1, \ldots, m$, such that $\xi+\sum_{i=1}^{m} \lambda_{i} \xi_{i}=0$. Therefore, $f(x)-f\left(x_{0}\right) \geqslant \xi^{T} \eta\left(x, x_{0}\right)+\sum_{i=1}^{m} \lambda_{i} \xi_{i}^{T} \eta\left(x, x_{0}\right)=$ $\left(\xi+\sum_{i=1}^{m} \lambda_{i} \xi_{i}\right)^{T} \eta\left(x, x_{0}\right)=0$.

REMARK. The point $x_{0}$ at which the Kuhn-Tucker conditions are satisfied is a local minimum of $(\mathrm{P})$ if $f$ and $g_{i}, i=1, \ldots, m$, are invex at $x_{0}$ for a common $\eta$, where invex at $x_{0}$ means that (2) is satisfied for all $x$ in a neighbourhood of $x_{0}$. The proof is essentially the same as above.

Hiriart-Urruty [13, Theorem 4.1] obtained improved necessary optimality conditions for $x_{0}$ to be a local minimum of $(P)$ by substituting

$$
0 \in \partial\left(f+\sum_{i=1}^{m} \lambda_{i} g_{i}\right)\left(x_{0}\right)
$$

for (5) with (6) and (7) unchanged. Theorem (d) implies that (5) is a stronger requirement for $x_{0}$ than (5), hence Theorem 5 holds when $\left(5^{\prime}\right)$ is substituted for (5). In fact, when $\left(5^{\prime}\right)$ is substituted for (5), we can weaken the invexity assumption in Theorem 5 .

THEOREM 6. Suppose $x_{0}$ is feasible for problem $(P)$ and Kuhn-Tucker conditions $\left(5^{\prime}\right),(6)$, and $(7)$ are satisfied at $x_{0}$. If $f+\sum_{i=1}^{m} \lambda_{i} g_{i}$ is invex on $X_{0}$, then $x_{0}$ is a global minimum for $(P)$.

Proof: Let $x$ be any other feasible point for (P); then by (6) and (7)

$$
\begin{aligned}
f(x)-f\left(x_{0}\right) & \geqslant f(x)+\sum_{i=1}^{m} \lambda_{i} g_{i}(x)-f\left(x_{0}\right)-\sum_{i=1}^{m} \lambda_{i} g_{i}\left(x_{0}\right) \\
& \geqslant\left(f+\sum_{i=1}^{m} \lambda_{i} g_{i}\right)^{0}\left(x_{0} ; \eta\left(x, x_{0}\right)\right) \\
& \geqslant 0, \quad \text { by }(5) .
\end{aligned}
$$

RemarK. The invexity assumption in Theorem 6 is weaker than the invexity assumption in Theorem 5. Indeed, if $f, g_{i}: X_{0} \rightarrow R$ are invex on $X_{0}$ for the same $\eta$, then since the lim sup of a sum is bounded above by the sum of the lim sups, for $y \in R^{m}$, $y \geqslant 0$, we have

$$
\begin{aligned}
f(x)+\sum_{i=1}^{m} y_{i} g_{i}(x)-f(u)-\sum_{i=1}^{m} y_{i} g_{i}(u) & \geqslant f^{0}(u ; \eta(x, u))+\sum_{i=1}^{m} y_{i} g_{i}^{0}(u ; \eta(x, u)) \\
& \geqslant\left(f+\sum_{i=1}^{m} y_{i} g_{i}\right)^{0}(u ; \eta(x, u))
\end{aligned}
$$


hence $\left(f+\sum_{i=1}^{m} y_{i} g_{i}\right)$ is invex on $X_{0}$.

Consider the following duals related to problem $(P)$ :

$\left(D_{1}\right)$

$$
\operatorname{Max} f(x)+\sum_{i=1}^{m} y_{i} g_{i}(x)
$$

$$
\text { such that } 0 \in \partial f(x)+\sum_{i=1}^{m} y_{i} \partial g_{i}(x) \text {, }
$$

$$
y_{i} \geqslant 0, i=1, \ldots, m
$$

and

$$
\operatorname{Max} f(x)+\sum_{i=1}^{m} y_{i} g_{i}(x)
$$

$$
\text { such that } 0 \in \partial\left(f+\sum_{i=1}^{m} y_{i} g_{i}\right)(x) \text {, }
$$

$$
y_{i} \geqslant 0, i=1, \ldots, m \text {. }
$$

By Theorem 2(d) the feasible region for $\left(D_{1}\right)$ is larger than the feasible region for $\left(D_{2}\right)$, hence the optimal value of the objective function in $\left(D_{1}\right)$ will be larger than the optimal value in $\left(D_{2}\right)$. Weak duality between $(P)$ and $\left(D_{1}\right)$ thus requires a stronger assumption than weak duality between $(P)$ and $\left(D_{2}\right)$.

THEOREM 7. (Weak Duality) (a) If for any fixed $y \in R^{m}, y \geqslant 0,\left(f+\sum_{i=1}^{m} y_{i} g_{i}\right)$ is invex on $X_{0}$, then weak duality holds between $(P)$ and $\left(D_{2}\right)$. (b) If $f$ and $g_{i}$, $i=1, \ldots, m$, are invex on $X_{0}$ for a common $\eta$, then weak duality holds for $(P)$ and $\left(D_{1}\right)$.

Proof: (a) Let $\bar{x}$ and $\left(x^{\prime}, y^{\prime}\right)$ be feasible for $(P)$ and $\left(D_{2}\right)$, respectively. Then

$$
\begin{aligned}
f(\bar{x})-f\left(x^{\prime}\right)-\sum_{i=1}^{m} y_{i}^{\prime} g_{i}\left(x^{\prime}\right) \geqslant & f(\bar{x})+\sum_{i=1}^{m} y_{i}^{\prime} g_{i}(\bar{x})-f\left(x^{\prime}\right)-\sum_{i=1}^{n} y_{i}^{\prime} g_{i}\left(x^{\prime}\right) \\
& \quad \text { since } y^{\prime} \geqslant 0 \text { and } \bar{x} \text { is feasible for }(\mathrm{P}) \\
\geqslant & \left(f+\sum_{i=1}^{m} y_{i}^{\prime} g_{i}\right)^{0}\left(x^{\prime} ; \eta\left(\bar{x}, x^{\prime}\right)\right) \\
\geqslant & 0, \text { since } 0 \in \partial\left(f+\sum_{i=1}^{m} y_{i}^{\prime} g_{i}\right)\left(x^{\prime}\right) .
\end{aligned}
$$


(b) Let $\bar{x}$ and $\left(x^{*}, y^{*}\right)$ be feasible for $(P)$ and $\left(D_{1}\right)$, respectively. Then

$$
\begin{array}{r}
f(\bar{x})-f\left(x^{*}\right)-\sum_{i=1}^{m} y_{i}^{*} g_{i}\left(x^{*}\right) \geqslant f(\bar{x})+\sum_{i=1}^{m} y_{i}^{*} g_{i}(\bar{x})-f\left(x^{*}\right)-\sum_{i=1}^{m} y_{i}^{*} g_{i}\left(x^{*}\right), \\
\text { since } y^{*} \geqslant 0 \text { and } \bar{x} \text { is feasible for }(\mathrm{P}), \\
\geqslant f^{0}\left(x^{*} ; \eta\left(\bar{x}, x^{*}\right)\right)+\sum_{i=1}^{m} y_{i}^{*} g_{i}^{0}\left(x^{*} ; \eta\left(\bar{x}, x^{*}\right)\right) ;
\end{array}
$$

since $\left(x^{*}, y^{*}\right)$ is feasible for $\left(D_{1}\right)$, there exist $\xi \in \partial f\left(x^{*}\right)$ and $\xi_{i} \in \partial g_{i}\left(x^{*}\right)$ such that $0=\xi+\sum_{i=1}^{m} y_{i}^{*} \xi_{i}$, hence

$$
\begin{aligned}
f^{0}\left(x^{*} ; \eta\left(\bar{x}, x^{*}\right)\right)+\sum_{i=1}^{m} y_{i}^{*} g_{i}^{0}\left(x^{*} ; \eta\left(\bar{x}, x^{*}\right)\right) & \geqslant \xi^{T} \eta\left(\bar{x}, x^{*}\right)+\sum_{i=1}^{m} y_{i}^{*} \xi_{i}^{T} \eta\left(\bar{x}, x^{*}\right) \\
& =\left(\xi+\sum_{i=1}^{m} y_{i}^{*} \xi_{i}\right)^{T} \eta\left(\bar{x}, x^{*}\right) \\
& =0 .
\end{aligned}
$$

Theorem 8. (Strong Duality) (a) Assume that $f+\sum_{i=1}^{m} y_{i} g_{i}$ is invex on $X_{0}$ for $y \in R^{m}, y \geqslant 0$. If $\bar{x}$ is optimal for $(P)$ and Kuhn-Tucker conditions (5'), (6) and (7) are satisfied at $(\bar{x}, \bar{y})$, then $\left(D_{2}\right)$ is maximised at $(\bar{x}, \bar{y})$ and the optimal values of $(P)$ and $\left(D_{2}\right)$ are equal. (b) Assume that $f$ and $g_{i}, i=1, \ldots, m$, are invex on $X_{0}$ for the same $\eta$. If $\bar{x}$ is optimal for $(P)$ and Kuhn-Tucker conditions (5)-(7) are satisfied at $(\bar{x}, \bar{y})$, then $\left(D_{1}\right)$ is maximised at $(\bar{x}, \bar{y})$ and the optimal values of $(P)$ and $\left(D_{1}\right)$ are equal.

Proof: (a) Since $(\bar{x}, \bar{y})$ satisfies $\left(5^{\prime}\right)$ and $(7),(\bar{x}, \bar{y})$ is feasible for $\left(D_{2}\right)$. By (6) and weak duality, $f(\bar{x})+\sum_{i=1}^{m} \bar{y}_{i} g_{i}(\bar{x})=f(\bar{x}) \geqslant f(x)+\sum_{i=1}^{m} y_{i} g_{i}(x)$, for all $(x, y)$ feasible for $\left(D_{2}\right)$. Thus $(\bar{x}, \bar{y})$ is optimal for $\left(D_{2}\right)$ and the optimal values of $(P)$ and $\left(D_{2}\right)$ are equal.

(b) In the proof of part (a), substitute (5) for $\left(5^{\prime}\right)$ and $\left(D_{1}\right)$ for $\left(D_{2}\right)$.

\section{REFERENCES}

[1] A. Ben-Israel and B. Mond, 'What is invexity?', J. Austral. Math. Soc. Ser. B 28 (1986), 1-9.

[2] F.H. Clarke, 'Generalized gradients of Lipschitz functionals', Adv. in Math. 40 (1981), 52-67. 
[3] F.H. Clarke, Optimization and nonsmooth analysis (Wiley, New York, 1983).

[4] B.D. Craven, 'Duality for generalized convex fractional programs', in Generalized concavity in optimization and economics, Editors S. Schaible and T. Ziemba, pp. 473-490 (Academic Press, 1981).

(5] B.D. Craven, 'Invex functions and constrained local minima', Bull. Austral. Math. Soc. 24 (1981), 357-366.

[6] B.D. Craven, 'Nondifferentiable optimization by smooth approximations', Optimization 17 (1986), 3-17.

[7] B.D. Craven and B.M. Glover, 'Invex functions and duality', J. Austral. Math. Soc. Series A 39 (1985), 1-20.

[8] R.R. Egudo and B. Mond, 'Duality with generalized convexity', J. Austral. Math. Soc. Ser. $B 28$ (1986), 10-21.

[8] M.A. Hanson, 'On sufficiency of the Kuhn-Tucker conditions', J. Math. Anal. Appl. 80 (1981), 545-550.

[10] M.A. Hanson and B. Mond, 'Further generalizations of convexity in mathematical programming', J. Inform. Optim. Sci (1982), 25-32.

[11] M.A. Hanson and B. Mond, 'Necessary and sufficient conditions in constrained optimization', Math. Programming 37 (1987), 51-58.

[12] J.B. Hiriart-Urruty, 'On optimality conditions in nondifferentiable programming', Math. Prog. 14 (1978), 73-86.

[13] J.B. Hiriart-Urruty, 'Refinements of necessary optimality conditions in nondifferentiable programming I', Appl. Math. Optim. 5 (1979), 63-82.

[14] V. Jeyakumar, 'Strong and weak invexity in mathematical programming', Methods Oper. Res. 55 (1985), 109-125.

[15] D.H. Martin, 'The essence of invexity', J. Optim. Theory Appl. 47 (1985), 65-76.

[16] R. Mifflin, 'Semismooth and semiconvex functions in constrained optimization', SIAM J. Control Optim. 15 (1977), 959-972.

[17] B. Mond and M.A. Hanson, 'On duality with generalized convexity', Mathematische $O p$ erationsforschung und Statistik Series Optimization 15 (1984), 313-317.

[18] B. Mond and T. Weir, 'Generalized concavity and duality', in Generalized Concavity in Optimization and Economics, Editors S. Schaible and T. Ziemba, pp. 473-490 (Academic Press, 1981).

[19] T.W. Reiland, 'A geometric approach to nonsmooth optimization with sample applications', Nonlinear Anal. 11 (1987), 1169-1184.

[20] E.M. Stern, Singular Integrals and Differentiability Properties of Functions: Princeton Mathematics Series 30 (Princeton University Press, Princeton, New Jersey, 1970).

Department of Statistics and

Graduate Program in Operations Research

Box 8203

North Carolins State University

Raleigh, NC 27695-8203

United States of America 